\title{
Masks in Medicine: Metaphors and Morality
}

\author{
Lindsey Grubbs ${ }^{1,2} \cdot$ Gail Geller $2,3,4$ (iD
}

Accepted: 30 December 2020 / Published online: 8 March 2021

(c) The Author(s), under exclusive licence to Springer Science+Business Media, LLC part of Springer Nature 2021

\begin{abstract}
We have never been so aware of masks. They were in short supply in the early days of COVID-19, resulting in significant risk to health care workers. Now they are highly politicized with battles about mask-wearing protocols breaking out in public. Although masks have obtained a new urgency and ubiquity in the context of COVID-19, people have thought about both the literal and metaphorical role of masks in medicine for generations. In this paper, we discuss three such metaphors - the masks of objectivity, of infallibility, and of benevolence - and their powerful role in medicine. These masks can be viewed as inflexible barriers to communication, contributing to the traditional authoritarian relationship between doctor and patient and concealing the authenticity and vulnerability of physicians. COVID masks, by contrast, offer a more nuanced and morally complex metaphor for thinking about protecting people from harm, authentic and trustworthy communication, and attention to potential inequities both in and beyond medical settings. We highlight the morally relevant challenges and opportunities that masks evoke and suggest that there is much to be gained from rethinking the mask metaphor in medicine.
\end{abstract}

Keywords Masks · Metaphor · Communication · Medical uncertainty $\cdot$ Social inequity Public health

We have never been so aware of masks. The early days of COVID-19 in the United States were marked by dire shortages of the personal protective equipment necessary to keep medical workers safe. N95 masks remain limited, precious resources that clinicians preserve in paper bags. At home, people stitch handmade masks or wrap scarves around their faces. In an increasingly open public sphere, some read masks as contributions to public health, as attempts to protect those around us. But masks have also become highly politicized, with fights breaking out in businesses, on streets, and in governmental bodies around

Gail Geller

ggeller@jhu.edu

1 California State University, East Bay, Oakland, CA, USA

2 Johns Hopkins University, Berman Institute of Bioethics, Baltimore, MD, USA

3 Johns Hopkins University School of Medicine, Baltimore, MD, USA

4 Johns Hopkins Bloomberg School of Public Health, Baltimore, MD, USA 
mask-wearing protocols. Such battles are exacerbated in part by an initial uncertainty surrounding the necessity of masks for the general public.

In medicine, there is profound discomfort with uncertainty. One colleague recently invoked "the mask" as a metaphor to critique medicine's desire to oversimplify complex phenomena and decision-making. Discussing the ethics of using SOFA (Sequential Organ Failure Assessment) scores and other numerical tools for allocating scarce resources (Frolic, Kata and Kraus 2009), he suggested that the value-laden nature of these calculations is disguised under a "mask of objectivity," which attributes simplified mathematical logic to highly subjective and emotionally fraught decisions. A quick search revealed that this evocative metaphor has been used before. Douard and Schultz (2013) invoke "the mask of objectivity" to describe the ways expert witnesses use neuroimaging in court cases on "psychopathy," obscuring the limits and technical interpretation inherent in neuroimaging by relying on the popular belief that such images provide pure scientific data.

Although masks have obtained a new urgency in the context of COVID-19, people have considered both the literal and metaphorical role of masks in medicine for generations. As a metaphor, masks are often deployed, as in the example above, to critique attitudes or habits that separate clinicians and patients. In 1975, Gorovitz and MacIntyre encouraged doctors to dispense with their "mask of infallibility" by acknowledging the inherent uncertainties in medicine. They attribute this mask not to arrogance but to physicians' belief that a posture of certainty protects patients from anxiety. In reality, they argue, by misconstruing a messy reality, this mask gives patients unreasonable expectations for seamless medical care, ultimately intensifying conflict around poor outcomes. Dispensing with this mask would require both physicians and patients to accept new forms of honest-and uncomfortable-communication about risk (Gorovitz and MacIntyre 1975).

Revisiting this metaphor, Jay Katz (2002) sees the "mask of infallibility" as contributing to the "traditional authoritarian relationship" between doctor and patient, a relationship that can damage both parties: "Masks can deceive not only the audience but the actor as well. The mask of infallibility makes it more difficult than it would otherwise be for physicians to explore their own doubts and uncertainties and precludes acknowledging them to patients" (199). Dispensing with the mask to reveal the true human face, Katz suggests, removes one barrier to a more equitable doctor-patient relationship. We must raise the "curtain of silence, of mistrust in patients' capacities to engage in conversation" (199). In Katz' formulation of the mask metaphor, then, the mask is a complete barrier between provider and patient, one in danger of disfiguring the clinician's actual face.

An even more dangerous metaphorical mask provides the title for psychologist Harlan Lane's Mask of Benevolence, in which the term signifies the paternalism of a medical field intent on "fixing" the Deaf community rather than accommodating them. Lane's metaphor indicts the medical profession: the "mask of benevolence" suggests a "face of malevolence" that must be hidden. In Lane's formulation, this mask "professes to serve deaf people" while actually "dominating, restructuring, and exercising authority over the deaf community" (1999, 43).

Lane's metaphor inspired a 2011 painting by Nancy Rourke, a Deaf artist. She depicts a crowd of people outlined in white against a black background with large Xs where the figures' faces should be and the phrase "Mask of Benevolence" scrawled across them. These Xs are blue, a color Rourke uses to denote discrimination against the Deaf. Only one of these figures - the most detailed and the only one with a discernable face-is circled in red, her color for empowerment and, with the only visible hands in the painting, signs the word "Stop" (Rourke 2011) For Rourke, then, the metaphor of the mask offers an effective 
means for critiquing discrimination and advocating for the empowerment of the Deaf community through inclusive communication (Fig. 1).

Metaphors have the power to structure our understanding and even behavior. All three of these metaphors - the masks of objectivity, of infallibility, of benevolence-evoke theatrical masks. These full-face affairs conceal the clinician's actual human face, replacing it with a false, immobile exterior. They are inflexible barriers to communication that may become dangerously fused to the face of the wearer. To wear a mask is to forsake authenticity or vulnerability.

The masks we are surrounded by today, though, are neither Halloween decorations nor theatrical equipment that cover one's true face with a plastic facsimile. Although obscuring the nose and mouth, the rest of the face remains visible, and most expressions can still be detected. While it is a kind of barrier, it is not a façade. What might it look like to reimagine these metaphors, turning away from theatrical masks to consider what insights are made possible by the images of the surgical mask, the N95, the home-stitched mask, or the bandana?

COVID masks bring ethical questions to the foreground, offering a more nuanced metaphor for thinking about moral considerations such as protecting people from harm, authentic and trustworthy communication, and attention to potential inequities both in and beyond medical settings. The shortage of masks poses an obvious and potentially fatal threat to medical professionals and patients. Outside of the medical setting, they create other dilemmas. Nehama Rogozen, a Deaf woman, writes about the difficult risk-benefit calculations she engages in when balancing the safety benefits of masks with the communication challenges posed by muffled speech and an inability to read lips (2020). Some say that clear masks should become standard in medical settings, which would improve communication not only with d/Deaf patients and physicians but with non-native English speakers who may benefit from visual cues. But these masks do not represent a simple solution to the

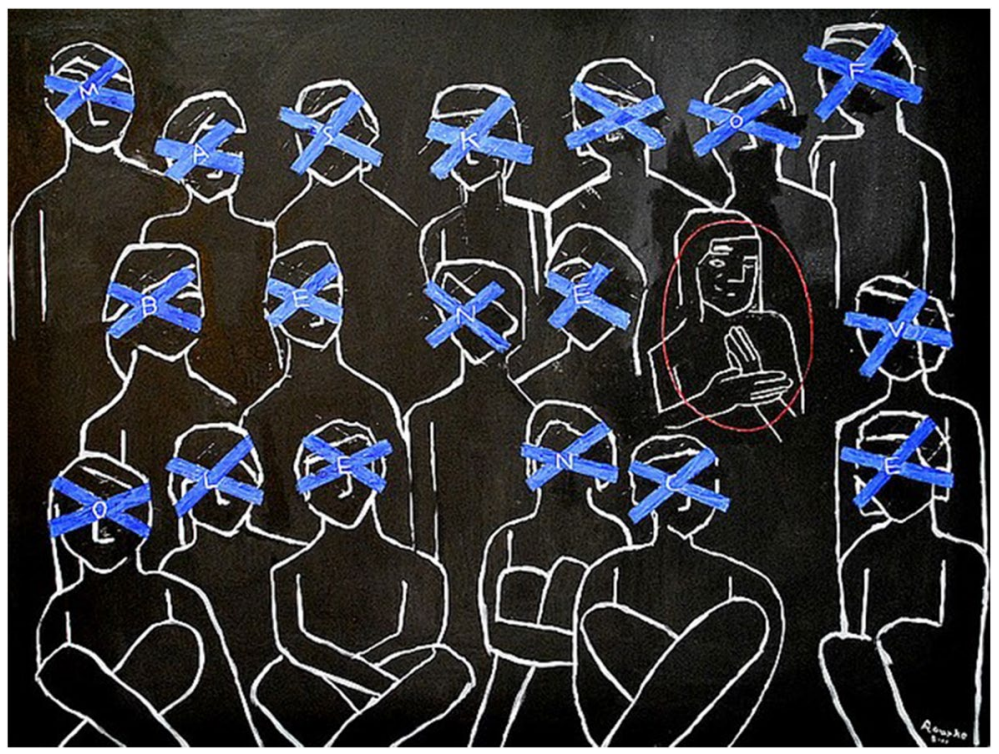

Fig. 1 Nancy Rourke, Mask of Benevolence, oil on canvas, 2011. https://www.nancyrourke.com/maskb enevolence.htm. 
communication conundrum. Rogozen, for example, sees these masks as "well-meaning" but not logistically feasible in daily life: would every person she encounters in a day have one? would she have to carry around a stash to hand out? The possibilities and challenges posed by adaptive masks demonstrate that, rather than a quick technological fix, we need a commitment to experimenting with a variety of communication strategies in order to provide individualized access for those who need it such as including physical printouts or dry-erase markers, texting, live captioning, ASL interpreters, or video chatting. No solution will work for everyone, and everyone needs to practice flexible, adaptive communication that attends to existing inequities in healthcare communication.

Broader societal inequities might also be exacerbated by mask-wearing. Gabriel Felix (2002) expresses his fear that as a Black doctor, wearing his mask in public could be lifethreatening. He knows that many people view Black men with suspicion, leading to potentially deadly encounters with law enforcement. So while masks are touted as a protective public health mechanism, they may paradoxically increase the risk of harm for Black people because of the realities of racism in the United States.

Today's ever-present masks evoke a range of morally relevant challenges and opportunities. They clarify how governmental decisions can leave medical staff in danger, highlight barriers to communication between clinicians and patients with disabilities, and expose the stakes of social and political injustice. At the same time, they reveal a shared vulnerability to the risks of infection, a commitment to protecting those we come in contact with, the need for creative communication strategies, and the urgency of racial justice movements.

Keeping this array of moral considerations simultaneously in mind requires tolerance for ambiguity. While the common metaphor of the theatrical mask in medicine suggests a binary state of affairs - one either wears a mask or shows their true face-the masks we see today require a commitment to constant renegotiation depending on context, forcing us to imagine new strategies of identification and communication that highlight the collaborative and experimental nature of human interaction. Removing today's masks would defeat their protective purpose but failing to work around their limitations would be morally inexcusable. For these reasons, we should rethink the mask metaphor in medicine. Rather than imagining a complete barrier between clinicians and patients, we can embrace an image that suggests mutual vulnerability and a commitment to developing workable solutions, one that asks us to examine our assumptions, cultivating tolerance for-and skill with managing - the uncertainty always inherent in medicine and life.

\section{References}

Douard, John, and Pamela Schultz. 2013. "The Mask of Objectivity: Digital Imaging and Psychopathy.” In Monstrous Crimes and the Failure of Forensic Psychiatry, International Library of Ethics, Law, and the New Medicine Series 53, 113-132. Dordrecht: Springer. https://doi.org/10.1007/978-94-007-5279-5_7.

Felix, Gabriel. 2020. "Wearing a Face Mask Helps Protect Me Against Covid-19, but Not Against Racism." STAT News. Accessed May 13, 2020. https://www.statnews.com/2020/05/13/black-man-think-twice -wearing-face-mask-in-public-racism/.

Frolic, Andrea, Anna Kata, and Peter Kraus. 2009. "Development of a Critical Care Triage Protocol for Pandemic Influenza: Integrating Ethics, Evidence and Effectiveness.” Healthcare Quarterly 12 (4): 54-62. https://doi.org/10.12927/hcq.2009.21054.

Gorovitz, Samuel, and Alasdair MacIntyre. 1975. "Toward a Theory of Medical Fallibility." Hastings Center Report 5 (6): 13-23.

Katz, Jay. 2002. The Silent World of Doctor and Patient. Baltimore: Johns Hopkins University Press.

Lane, Harlan. 1999. The Mask of Benevolence: Disabling the Deaf Community. San Diego: DawnSignPress. 
Rogozen, Nehama. 2020. "I'm Deaf and I Lip-Read. All Those Masks are Presenting a Problem." Slate. May 12. https://slate.com/human-interest/2020/05/deaf-masks-lip-reading-coronavirus.html.

Rourke, Nancy. 2011. Mask of Benevolence. Nancy Rourke Paintings website. Accessed June 29, 2020. http://www.nancyrourke.com/maskbenevolence.htm.

Publisher's Note Springer Nature remains neutral with regard to jurisdictional claims in published maps and institutional affiliations. 